\title{
Active vitamin D supplementation and COVID-19 infections: review
}

\author{
Nakhoul Farid ${ }^{1,2,3}$ (D) Nakhoul Rola $^{4} \cdot$ Elias A. T. Koch ${ }^{5} \cdot$ Nakhoul Nakhoul $^{6}$ \\ Received: 25 September 2020 / Accepted: 1 December 2020 / Published online: 6 January 2021 \\ (C) Royal Academy of Medicine in Ireland 2021
}

\begin{abstract}
SARS-CoV-2, causing the lethal disease COVid-19, is a public health emergency in the 2020 global pandemic. The outbreak and fast spreading of SARS-CoV-2 have a high morbidity and mortality specifically in elder patients with chronic diseases such as diabetes mellitus, arterial hypertension, chronic kidney disease, and organ transplanted patients with immunosuppressive therapy. Preliminary results support different treatments such as chloroquine and convalescent plasma infusion in severe cases, with good outcome. On the other hand, the efficacy of supplementation with active vitamin $\mathrm{D}$, an immunomodulator hormone with antiinflammatory and antimicrobial effects, is unproven. A recent study reported that vitamin D attains antiviral effects, via blocking viral replication directly. SARS-CoV-2 primarily uses the immune evasion process during infection via the envelope spike glycoprotein, which is followed by a cytokine storm, causing severe acute respiratory disease syndrome and death. SARS$\mathrm{CoV}-2$, by using the well-known angiotensin-converting enzyme 2 by the protein spike, as the host receptor to enter into alveolar, myocardial, and renal epithelial cells, can be disrupted by vitamin D. However, the correlation between vitamin D levels and COVID-19 deaths in previous studies was insignificant. Retrospective studies demonstrated a correlation between vitamin D status and COVID-19 severity and mortality, while other studies did not find this correlation. Studies have shown that, vitamin D reduces the risk of acute viral respiratory tract infections and pneumonia via direct inhibition of viral replication, antiinflammatory and immunomodulatory effects. The data available today regarding the beneficial protective effect of vitamin $\mathrm{D}$ is unclear and with conflicting results. Large randomized control trials are necessary to test this hypothesis. In this review, we will explain the cross talk between the active vitamin $\mathrm{D}$ and the angiotensin-converting enzyme 2 , and summarize the data from the literature.
\end{abstract}

Keywords Angiotensin converting enzyme $2 \cdot$ COVID-19 $\cdot$ Renin $\cdot$ SARS-CoV-2 $\cdot$ Vitamin D

\section{Abbreviation}

ACE2 Angiotensin converting enzyme 2

Nakhoul Farid

fnakhoul@poria.health.gov.il; fnakhoul60@gmail.com

1 Nephrology and Hypertension Division, Baruch Padeh Poriya medical Center, Erlangen, Germany

2 Diabetes and Metabolism Lab, Baruch Padeh Poriya Medical Center, Erlangen, Germany

3 Azrieli Faculty of Medicine, Bar Ilan University, Ramat-Gan, Israel

4 Department of Anaesthesiology, University Hospital Erlangen, Erlangen, Germany

5 Department of Dermatology, University Hospital Erlangen, Erlangen, Germany

6 Ophtalmology Division, Baruch Padeh Poriya Medical Center, Ramat-Gan, Israel
VDR Vitamin D receptor

RAAS Renin angiotensin-aldosterone system

AII Angiotensin II

AI Angiotensin I

\section{Introduction}

SARS-CoV-2, the novel coronavirus, causes the serious COVID-19 disease, a highly contagious and wide spreading, worldwide disease. SARS-CoV-2 is particularly pathogenic in humans and is associated with high morbidity and mortality. Coronavirus disease (COVID-19) represents the causative agent of a potentially fatal disease if not treated early and aggressively. Angiotensin-converting enzyme 2 (ACE2) has been established as the functional host receptor for severe acute respiratory syndrome coronavirus 2 (SARS-CoV-2). Altered ACE2 expression in different epithelial cells, 
especially alveolar cells, can influence the COVID-19 severity and progression.

Many factors could have a role in the high morbidity and mortality in COVID-19, including advanced age (elderly people $>70$ years old); sex; chronic diseases such as obesity, arterial hypertension, diabetes mellitus, and chronic airway disease; quality of healthcare system; and socioeconomic status [1-4]. Due to the severity and the fatal outcome in some patients, FDA, recently permitted the use of remdesivir for severe COVID-19. Remdesivir, a broad-spectrum antiviral agent, is in use now in severe cases in hospitalized patients. In addition, it can be used in combination with dexamethasone, chloroquine, and convalescent plasma infusion, shortening the admission period and the use of mechanical ventilation. Study results on the use of active vitamin D compound are encouraging [5].

Vitamin D undergoes hydroxylation by the 1- $\alpha$-hydroxylase in the proximal convolute tubule cells of the kidney, to yield its active form $1,25(\mathrm{OH})_{2} \mathrm{D} 3$. The active $1,25(\mathrm{OH})_{2} \mathrm{D} 3$ binds to the intracellular vitamin $\mathrm{D}$ receptor (VDR) to activate vitamin D response elements within target genes [6]. In the kidney, vitamin $\mathrm{D}$ is important for maintaining podocyte proteins nephrin-podocin, and suppressing renin gene expression and inflammation. Inverse correlation was found between low vitamin D levels and infection risk such as influenza. Vitamin D insufficiency affects the immune function, active vitamin D exerts an immunomodulation role, increasing natural immunity by secretion of antiviral peptides which improves mucosal defense. Recently, different studies support the concept that the relative vitamin D status in the general population could influence the outcome of COVID-19.

In the literature, supplementation of vitamin $\mathrm{D}$ or its active derivatives has shown inconclusive benefit results. Others have demonstrated a reduced endothelial and epithelial cell injury, in COVID-19 patients especially in older people with chronic diseases, with vitamin D supplementation $[7,8]$.

\section{Active vitamin D (calcifedol)—RAAS axis crosstalk}

The active vitamin D, 1,25-dihydroxycholecalciferol, plays an essential role in the immune system. Active vitamin D interferes with the majority of the immune system cells such as B and T lymphocytes, which express VDR. Vitamin D can induce the human cathelicidin gene expression by binding to the vitamin D responsive element (VDRE) in various tissues, such as the respiratory epithelium, myocytes, and renal cells, with increased synthesis of cathelicidin. Cathelicidin has shown antimicrobial activity against most viruses, including corona viruses. Furthermore, vitamin D inhibits the production of proinflammatory cytokines and increases the production of anti-inflammatory cytokines [8-11].
Vitamin D deficiency especially in the elderly with chronic diseases such as diabetes mellitus, arterial hypertension, obesity, and chronic kidney disease, is a public health problem worldwide. Vitamin D exerts an immuno-modulation role, increasing natural immunity by secretion of antiviral peptides, which improves mucosal defenses, interferon $\gamma$, tumor necrosis factor $\alpha(\mathrm{TNF} \alpha)$ and regulating the immune system through inhibiting $\mathrm{T}$ helper cell type 1 responses and stimulating T cells $[8,9]$. People with low active vitamin D blood levels tested positive for COVID-19 more than people with normal levels.

Clinical studies had shown that low levels of serum vitamin D were associated with acute respiratory tract infections including epidemic influenza. The benefit of active compounds of vitamin D, especially paricalcitol, that selectively bind VDR in different cells, with well-established inhibitory effect on cell proliferation and fibrosis, was previously proven. Several studies have shown that low vitamin D blood levels can increase plasma renin activity, and consequently the renin-angiotensin-aldosterone-system (RAAS) activity. The same applies to the decreasing renin activity with increasing vitamin D levels. There is an inverse relationship between circulating vitamin $\mathrm{D}$ and renin, which is explained by the fact that vitamin $\mathrm{D}$ is a negative regulator of renin expression by suppressing the renin gene promoter, thus acting as a negative RAAS regulator. The $1,25(\mathrm{OH})_{2} \mathrm{D} 3$ induced repression of the renin gene expression is independent from angiotensin II (Ang II) feedback regulation. People with vitamin D deficiency can express an increase in the renin levels with consequent increased Ang II formation and harmful negative effects via angiotensin II type I receptor [12].

Angiotensin-converting enzyme 2 (ACE2) was established as the functional host receptor for SARS-CoV-2, and plays a crucial role in the pathogenesis of COVID-19, as the main viral entry into human cells and replication. Angiotensin II (Ang II) is a natural peptide hormone in the RAAS with strong vasoconstriction effect. ACE2 directly catalyze Ang II, thereby lowering its levels. COVID-19 infection downregulates ACE2, which in turn could lead to excessive accumulation of Ang II. High levels of Ang II may cause ARDS, myocarditis with heart failure and acute kidney injury [13, 14] (Fig. 1).

\section{Coronavirus disease (COVID-19)}

COVID-19 is cause by SARS-CoV-2 that affects the lower respiratory tract and manifests as severe pneumonia in humans. COVID-19 is a serious contagious illness in the elderly and patients with chronic illness, with very strong spread. Most SARS-CoV-2 infected patients have developed mild symptoms, but the majority of cases have developed various fatal complications including organ failure, vascular thrombosis, severe pneumonia, and acute respiratory distress 


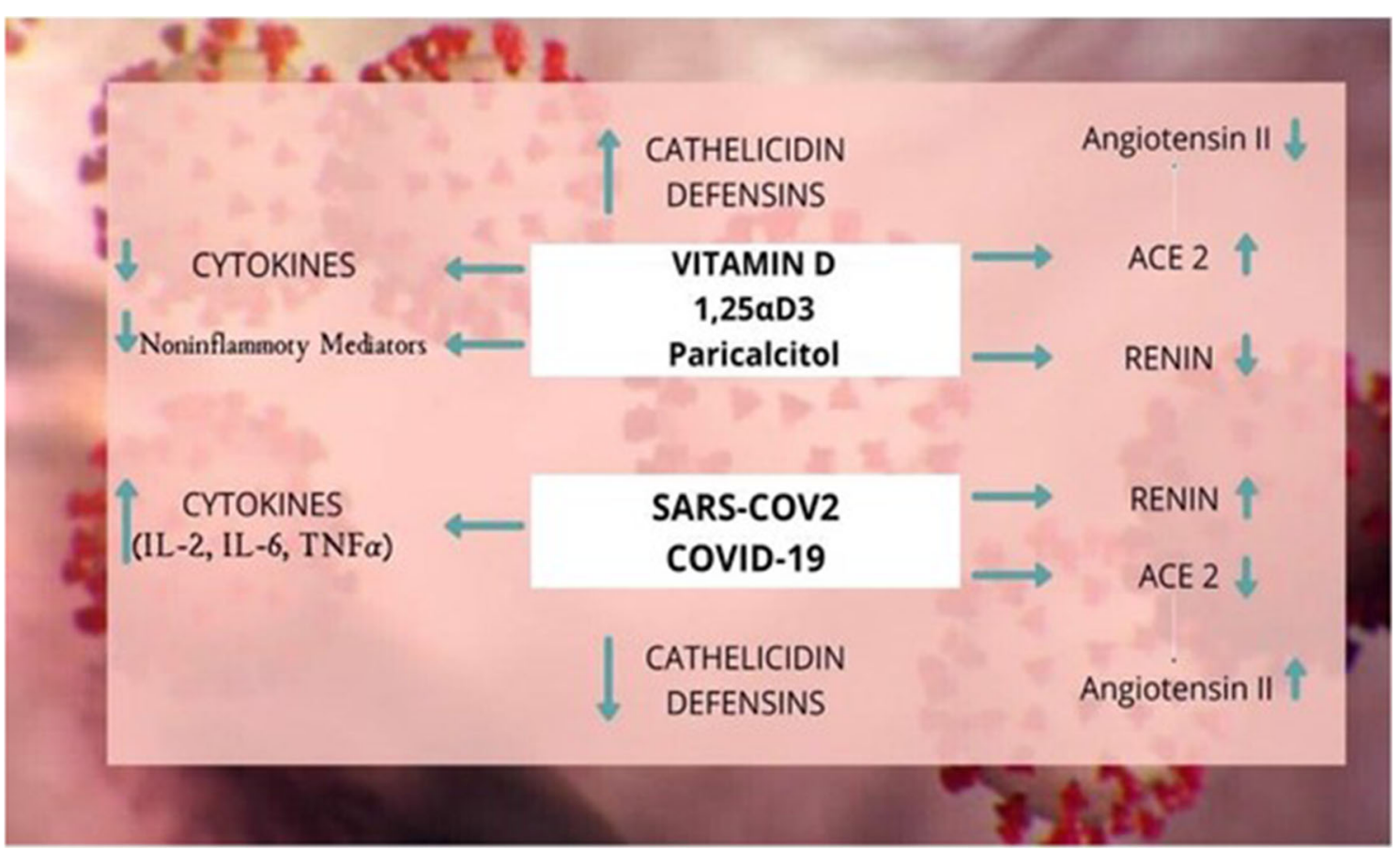

Fig. 1 Schematic presentation on the interaction between vitamin D and SARS-COV2 on inflammatory process and RAAS/ACE2

syndrome if not supported by immediate treatment. Fifty percent of those infected with RS-CoV-2 are male with a median age of 50-60 years old, but in the last month, young persons are also affected. Patients who required intensive care support were older and had multiple comorbidities including cardiovascular, cerebrovascular, endocrine, digestive, and chronic respiratory disease $[15,16]$.

Angiotensin-converting enzyme 2 (ACE2) is the main host cell receptor for SARS-CoV-2 by envelope spike glycoprotein [17]. ACE2 is express in alveolar cells of lungs, renal proximal convolute cells, vascular cells, and myocytes. The higher the expression of the enzyme, the higher is the development of the acute respiratory distress syndrome (ARDS) with pneumonitis and acute kidney injury with failure. This syndrome is associated with a cytokine storm, which trigger $\mathrm{T}$ helper 1 (Th1) cell response and high release of interleukins [17]. Therefore, in some centers, dexamethasone is recommended as early treatment to block the cytokine storm, and later antiviral treatment such as remdesivir and convalescent plasma infusion.

\section{Vitamin D supplementation is helpful in SARS-CoV-2?}

COVID-19 infection can be treated in different manners including anti-viral agents and plasma transfusion. Extensive evidence has linked vitamin D deficiency to greater risk of infection, particularly from respiratory diseases like COVID-19 [18-20]. The active vitamin D and his receptor (VDR) supports production of antimicrobial peptides such as cathelicidin and defensins, especially in the respiratory alveolar epithelium. Other pathway used by vitamin $\mathrm{D}$ to protect from infection is to reduce the inflammatory response to infection with SARSCoV-2 [21]. Vitamin D is known to interact with a protein in the pathway angiotensin-converting enzyme 2 (ACE2), which is also used by SARS-CoV-2 as an entry receptor. While SARS-CoV-2 downregulates expression of ACE2, vitamin D promotes expression of this gene [17].

SARS-CoV-2 not only reduces the ACE2 expression but also leads to further limitation of the ACE2/Ang 1-7/Mas' axis via ADAM17 activation, which in turn promotes the entry of the virus into the cell. This results in an increase in Ang II, which further upregulates ADAM 17. This process may contribute to lung damage, myocardial injury with heart failure and vessel damage with fata thrombosis, which observed in severe COVID-19 patients. Active vitamin D can interfere with ACE2-angiotensin 1-7-ADAM17 to promote ACE2 synthesis and down regulation of AII synthesis [22].

\section{Conclusions and recommendations}

Retrospective studies demonstrated a correlation between vitamin D and COVID-19 cases and outcomes, while other studies did not find any correlation. Yet, there is insufficient evidence on the association between vitamin $\mathrm{D}$ levels and COVID-19 severity and mortality. The active vitamin D, can exert a pulmonary, renal, and cardiac protective effect in terms of reducing inflammation and fibrosis by modulating the 
immune function through effects on T cells. Higher vitamin D levels correlate with lower interleukin 6 levels, which are a major target for controlling cytokine storm in COVID-19 [23-26]. There is no doubt that proper vaccine and selective antibodies from the plasma of convalescing patients, is the most appropriate treatment $[27,28]$.

Acknowledgments This work was supported by Karim Family in memory of their son Hasan Karim Magd Alkoroum, Israel.

Authors' contributions F.N. and R.N. drafted the manuscript. N.N. and E.A.T.K provided critical analysis to the paper. All authors read and approved the final manuscript.

Funding MIGAL, the Internal ministry for the development of GalileeNorth Israel.

\section{Compliance with ethical standards}

Conflict of interest The authors declare that they have no conflict of interest.

\section{References}

1. Singhal T (2020) A review of Coronavirus Disease-2019 (COVID19). Indian J Pediatr 87(4):281-286

2. Sifuentes-Rodríguez E, Palacios-Reyes D (2020) COVID-19: the outbreak caused by a new coronavirus. Bol Med Hosp Infant Mex 77(2):47-53

3. Rothan HA, Byrareddy SN (2020) The epidemiology and pathogenesis of coronavirus disease (COVID-19) outbreak. J Autoimmun 109:102433

4. Berlin DA, Roy M, Gulick RM, Martinez FJ (2020) Severe Covid19. NEJM

5. Beigel OH, Tomashek KM, Dodd LE, et al. (2020) for the ACTT-1 study group members. Remdesivir for the treatment of Covid-19 preliminary report. NEJM, May.

6. Borel P, Caillaud D, Cano NJ (2015) Vitamin D bioavailability: state of the art. Crit Rev Food Sci Nutr 55(9):1193-1205

7. Mitchell F (2020) Vitamin-D and COVID-19: do deficient risk a poorer outcome? Lancet Diabetes Endocrinol. 8(7):570

8. Nurshad A (2020) Role of vitamin D in preventing of COVID-19 infection, progression and severity. J Infect Public Health. 20;S1876-0341(20)30531-1

9. Cantorna MT, Snyder L, Lin YD et al (2015) Vitamin D and 1, 25(OH)2D regulation of T cells. Nutrients 7(4):3011-3021

10. Chun RF, Liu PT, Modlin RL et al (2014) Impact of vitamin D on immune function: lessons learned from genome-wide analysis. Front. Physiol. 5:151

11. Baeke F, Takiishi T, Korf H, Gysemans C, Mathieu C (2010) Vitamin D: modulator of the immune system. Curr Opin Pharmacol. (4):482-496

12. Giménez VMM, Sanz RL, Marón FJM, Ferder L, Manucha W (2020) Vitamin D-RAAS connection: an integrative standpoint into cardiovascular and neuroinflammatory disorders. Curr Protein Pept Sci. 21(Jun 6)

13. Simões e Silva AC, Silveira KD, Ferreira AJ et al (2013) ACE2, angiotensin-(1-7) and Mas receptor axis in inflammation and fibrosis. Br J Pharmacol. 169(3):477-492

14. Reynolds HR, Adhikari S, Pulgarin C (2020) Renin-angiotensinaldosterone system inhibitors and risk of Covid-19. N Engl J Med. 382:2441-2448

15. Kannan S, Shaik Syed AP, Sheeza A et al (2020) COVID-19 (Novel Coronavirus 2019) - recent trend, review. Eur Rev Med Pharmacol Sci. 24(4):2006-2011

16. Ackermann M, Verleden SE, Kuehnel M, Haverich A, Welte T, Laenger F, Vanstapel A, Werlein C, Stark H, Tzankov A, Li WW, Li VW, Mentzer SJ, Jonigk D (2020) Pulmonary vascular endothelialitis, thrombosis, and angiogenesis in Covid-19. N Engl J Med. 383:120-128

17. Bourgonje AR, Abdulle AE, Timens W et al. (2020) Angiotensinconverting enzyme 2 (ACE2), SARS-CoV-2 and the pathophysiology of coronavirus disease 2019 (COVID-19). J Pathol.

18. Zemb P, Bergman P , Camargo CA Jr et al (2020) Cavalier E, Cormier C, Courbebaisse M, et al. Vitamin D deficiency and the COVID-19 pandemic. J Glob Antimicrob Resist. 22:133-134

19. Biesalski HK (2020) Vitamin D deficiency and co-morbidities in COVID-19 patients - a fatal relationship? Nfs Journal 20:10-21

20. Carpagnano GE, Di Lecce V, Quaranta VN et al (2020) Vitamin D deficiency as a predictor of poor prognosis in patients with acute respiratory failure due to COVID-19. J Endocrinol Invest 9:1-7

21. Grant WB, Lahore H, McDonnell SL et al (2020) Evidence that vitamin D supplementation could reduce risk of influenza and COVID-19 infections and deaths. Nutrients 12(4):988

22. Mendoza A, Lazartigues E (2015) The compensatory reninangiotensin system in the central regulation of arterial pressure: new avenues and new challenges. Ther Adv Cardiovasc Dis 9(4): 201-208

23. Martineau AR (2017) Vitamin D supplementation to prevent acute respiratory tract infections: systematic review and meta-analysis of individual participant data. BMJ. 356:6583-6594

24. Weir EK, Thenappan T, Bhargava M et al (2020) Does vitamin D deficiency increase the severity of COVID-19? Clin Med (Lond) Jul 20(4):e107-e108

25. Ahn DG, Shin HJ, Kim MH et al (2020) Current status of epidemiology, diagnosis, therapeutics, and vaccines for novel coronavirus disease 2019 (COVID-19). J Microbiol Biotechnol 30(3):313-324

26. Meltzer DO, Best TJ, Zhang $\mathrm{H}$ et al (2020) Original investigation. Infectious diseases association of vitamin D status and other clinical characteristics with COVID-19 test results. JAMA Network Open 3(9):e2019722

27. Shanmugaraj B, Siriwattananon K, Wangkanont K (2020) Perspectives on monoclonal antibody therapy as potential therapeutic intervention for Coronavirus disease-19 (COVID-19). Asian Pac J Allergy Immunol 38(1):10-18

28. Jackson LA, Anderson EJ, Rouphael NG et al (2020) An mRNA Vaccine against SARS-CoV-2 — preliminary report, for the mRNA-1273 Study Group. NEJM:14

Publisher's note Springer Nature remains neutral with regard to jurisdictional claims in published maps and institutional affiliations. 